Reprod. Nutr. Dévelop., 1982, 22 (3), 511-522.

\title{
Répartition des concentrations enzymatiques dans la muqueuse abomasale du veau préruminant. Evolution avec l'âge
}

\author{
P. GUILloteAu, R. DELANSORNE (*), R. TOULLEC
}

avec la collaboration technique de Marguerite BEAUFILS, S. BOUSSION et J. N. NOUCHET

Station de Recherches zootechniques, I.N.R.A. 65, rue de Saint-Brieuc, 35042 Rennes Cédex

${ }^{*}$ ) Chaire $d^{\prime}$ Anatomie pathologique et $d^{\prime}$ Histologie Ecole Nationale Vétérinaire

7, avenue du Général de Gaulle, 94704 Maisons-Alfort

Summary. - Distribution of enzyme concentrations in the abomasal mucosa of the preruminant calf. Age changes.

The aim of this trial was to draw an "enzyme map " of the abomasum of the preruminant calf in order to determine the secretory capacities of various areas and to study their age changes. Three pairs of calves were slaughtered at 1.5 days and at 10 and 19 weeks. The abomasum was divided into 12 parts (fig. 1). The chymosin and pepsin extracted from each part were separated and assayed.

We found large individual variations in the amount and distribution of the enzymes (fig. 2, table 1) but, in all cases, more than 78 p. 100 were present in the fundus, while the antrum usually contained very little. The cardiac part contained as much as the fundic parts of similar size. The distribution of enzymes in the fundus between the parietal and visceral surfaces or between the proximal, median and distal plicae did not seem to change with age. On the contrary, that part of the enzymes provided by the ventral surface (along the greater curvature) increased with age, while the part provided by the dorsal surface (along the lesser curvature) decreased (fig. 2 ; table 2). However, the supply of the distal region (parts $3+7$ ) of the latter surface varied within much smaller limits, and the pepsin/chymosin ratio remained close to that observed in the whole abomasum. Therefore, the distal region would be the most suitable for making an abomasal pouch which would retain the same representativeness in relation to the whole of the abomasum. It could eventually be extended on the side of the distal plica.

\section{Introduction.}

Les sécrétions de la caillette du veau peuvent être étudiées en collectant le suc gastrique produit par une poche abomasale réalisée dans la zone fundique (Ash, 1961 ; Hill et Gregory, 1951 ; Grosskopf, 1959 ; Rusev, Petrov et Bocheva, 1971 ; Guilloteau et Le Calve, 1977 ; Williams, Roy et Gillies, 1976). II est néanmoins très difficile de connaître la proportion de suc gastrique obtenu à partir d'une poche, par rapport à la sécrétion abomasale totale. II est possible 
d'évaluer les « potentialités » de sécrétion d'enzymes (chymosine et pepsine), en les dosant avec leurs proenzymes, dans la muqueuse de la poche et dans celle du reste de la caillette ; cependant, ces mesures ne procurent qu'une image instantanée.

Andren, Bjoerck et Claesson (1980) ont montré qu'à différents âges et avec divers régimes alimentaires, les concentrations en mடynles sont toujours plus élevées dans le fundus que dans l'antrum ; mais, bien qu'ils aient découpé le fundus en 4 portions, leurs résultats ne permettent pas d'estimer l'évolution des concentrations le long de la grande et de la petite courbure. II nous a donc paru intéressant de dresser úne " cartographie enzymatique " détaillée de la caillette, pour déterminer les capacités de sécrétion des diverses zones et étudier leur évolution avec l'âge. Une étude histologique est menée parallèlement sur d'autres animaux ; cet aspect sera rapporté dans une publication ultérieure.

\section{Matériel et méthodes.}

Animaux et aliments. - L'expérience porte sur 6 veaux de race Frisonne. Les veaux I et II sont sacrifiés un jour et demi après la naissance après avoir reçu deux repas de colostrum. Les autres veaux reçoivent d'abord le colostrum puis un lait de remplacement dont les protéines proviennent du lait ; ils restent au stade préruminant jusqu'à leur abattage qui a lieu à l'âge de 10 semaines pour les veaux III et IV et de 19 semaines pour les veaux $\mathrm{V}$ et VI. Au moment de I'abattage, les poids vifs des animaux I à VI sont respectivement de 40,35, 103, 100,179 et $182 \mathrm{~kg}$.

Prélèvement. - Aussitôt après l'abattage, la caillette est prélevée et recouverte de glace pilée pour être transportée au laboratoire où elle est vidée de son contenu et ouverte selon la petite courbure. Elle est ensuite dégraissée et lavée avec du sérum physiologique réfrigéré et additionné de thymol. Elle est alors étalée sur une plaque de verre réfrigérée par de l'alcool froid $\left(-18^{\circ} \mathrm{C}\right)$ et découpée en 12 portions (fig. 1). Les sections précises proximale et distale correspondent respectivement à la fin de l'épithélium kératinisé des préestomacs et au début de la muqueuse duodénale, juste à la base du torus pylorique. La ligne fictive qui joint les naissances des plis spiraux sépare la zone orificielle de la zone fundique. Cette dernière se délimite ensuite de la zone antrale par le changement morphologique net du revêtement interne qui passe du gris rougeâtre au jaune rosé. Le schéma de découpe de la zone fundique est conçu de façon à obtenir dix portions ayant approximativement la même taille, en respectant une symétrie par rapport à la grande courbure. Le numérotage est tel que les portions impaires soient dorsales et les portions paires ventrales (sauf pour 1 et 10). Les portions 1 à 5 forment la face gauche ou viscérale et les portions 6 à 10 correspondent à la face droite ou pariétale.

Pour chacun des douze fragments obtenus, la muqueuse est séparée du reste de la paroi abomasale (musculeuse et séreuse) selon le plan de clivage naturel que constitue la sous-muqueuse. Le prélèvement ainsi recueilli est pesé et 


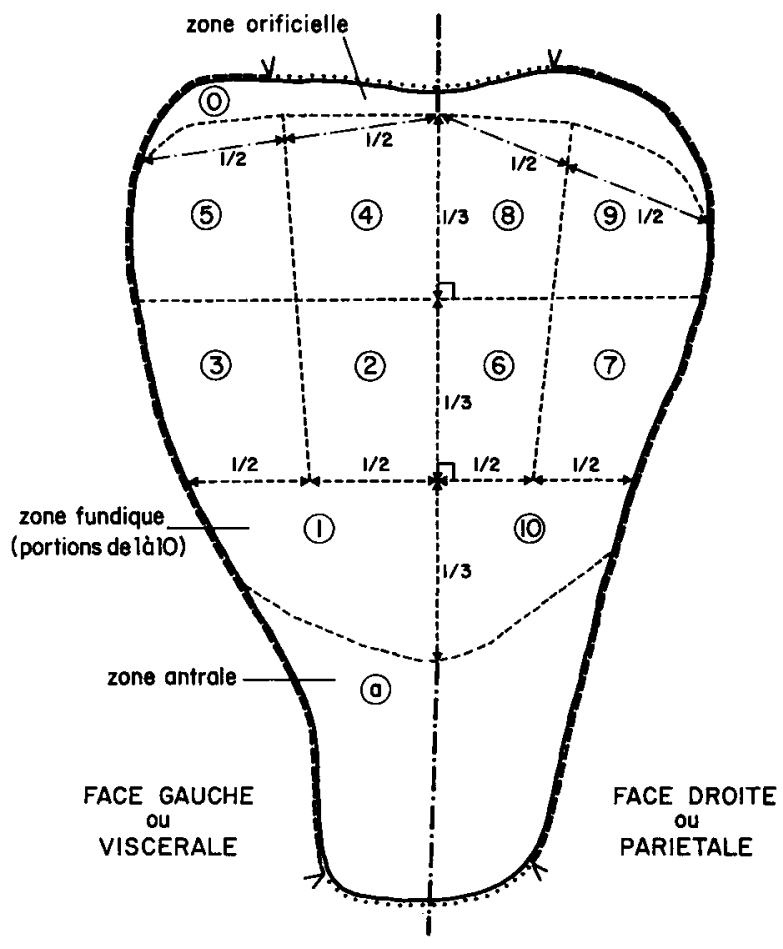

FIG. 1. - Représentation du plan de découpe de la caillette (ouverte le long de la petite courbure et étalée, la muqueuse vers le haut).

ligne de découpe :

orifices ;
- - petite courbure ;

$-\cdot \cdot-$ grande courbure.

immédiatement congelé dans l'azote liquide. II s'écoule environ $1 \mathrm{~h}$ entre l'abattage de l'animal et la congélation. Une partie de la sóus-muqueuse reste accolée à la muqueuse; du fait de sa faible importance lépaisseur résiduelle minime, tissu conjonctif lâche), nous assimilerons le poids obtenu au poids de la muqueuse.

Analyses. - Dès la sortie du congélateur, la muqueuse abomasale est découpée au scalpel et immédiatement broyée dans un récipient entouré de glace pilée, à l'aide d'un omni-mixer Sorval. Elle subit ensuite trois extractions à l'aide de 2 fois son poids de solution aqueuse de chlorure de sodium à 12 p. 100 maintenue à $10^{\circ} \mathrm{C}$. Les extraits sont chromatographiés sur une colonne de DEAE-cellulose selon la technique de Garnot et al. (1972). Trois activités enzymatiques (totale, chymosique, pepsique) sont déterminées par la mesure du temps de coagulation d'une solution de caséine $\kappa$ à $0,2 p$. 100, selon la méthode de Douillard et Ribadeau-Dumas (1970). Les résultats sont d'abord exprimés en $\kappa U$, unités arbitraires correspondant à l'inverse du temps de coagulation, puis 
transformés en poids d'enzymes (exprimés en $\mathrm{mg}$ ) après étalonnage de la préparation de caséine $\kappa$ utilisée. Nous distinguons le contenu enzymatique qui est la quantité d'enzymes (en $\mathrm{mg}$ ) extraites et dosées dans une partie de muqueuse, de la concentration, qui est ce contenu enzymatique rapporté au gramme de muqueuse correspondante.

\section{Résultats.}

Chez tous les animaux, la concentration en pepsine de la muqueuse est beaucoup plus élevée (de 2,0 à 7,5 fois) dans la zone fundique que dans la zone antrale (tabl. 1). Il en est de même pour la concentration en chymosine (de 2,2 à 13,6 fois), sauf chez le veau III où elle est du même ordre dans les 2 zones ; la zone fundique de cet animal est également la moins riche en chymosine $10,9 \mathrm{mg} / \mathrm{g}$ de muqueuse au lieu de 2,3 à 7,8$)$. La zone antrale ne renferme donc généralement qu'une part très faible (de 1 à $5 \mathrm{p}$. 100) des contenus enzymatiques totaux, par rapport à son importance pondérale (de 6 à 20 p. 100), excepté chez le veau III où elle apporte 16 p. 100 de la chymosine pour $15 \mathrm{p} .100$ du poids total de muqueuse. Dans tous les cas, la zone fundique est nettement prépondérante puisqu'elle fournit de 79 à 94 p. 100 des contenus enzymatiques totaux. La zone orificielle est toujours similaire, en concentration comme en contenu, à une onzième portion de type fundique (tabl. 1 et fig. 2). Aucune portion ne présente de rapport chymosine/pepsine systématiquement supérieur ou inférieur à la moyenne (tabl. 1). Pour la totalité de la caillette, ce rapport varie largement (de 2,1 à 19,5), mais le nombre d'animaux est insuffisant pour mettre en évidence une influence de l'âge.

Les portions fundiques peuvent être regroupées en différentes régions topographiques (tabl. 2). La répartition des contenus enzymatiques entre les

FIG. 2. - Diagramme de la cartographie enzymatique de la caillette chez les 6 veaux.

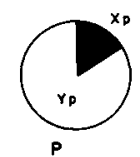

$P=$ Numéro ou initiale de la portion de découpe (voir fig. 1). $X_{P}=$ Quantité de pepsine présente dans la portion $P$ len p. 100 de la quantité totale de pepsine présente dans la caillette) D'où $\sum_{P} X_{P}=100 . Y_{P}=$ Quantité de chymosine présente dans la portion $P$ len $p .100$ dé la quantité totale de chymosine présente dans la caillette). D'où $\sum_{P} Y_{P}=100$.

Pour chaque portion $\mathrm{P}$ : - La surface du cercle est proportionnelle à la quantité d'enzymes (chymosine + pepsine) présente dans la portion $P$ par rapport à la quantité totale d'enzymes (chymosine + pepsine) présente dans la caillette (p. 100). - La surface $\mathrm{SX}_{\mathrm{p}} \mathbf{D}$ est proportionnelle à la quantité de pepsine présente dans la portion $P$ par rapport à la quantité totale d'enzymes (chymosine + pepsine) présentes dans la portion $P$. - La surface $S Y_{p} \square$ est proportionnelle à la quantité de chymosine présente dans la portion $P$ par rapport à la quantité totale d'enzymes (chymosine + pepsine) présentes dans la portion $P$. $-D^{\prime}$ où $\sum_{P}\left(S X_{P}+S Y_{P}\right)=100$. Ainsi, les diagrammes des différentes caillettes étudiées sont comparables. 

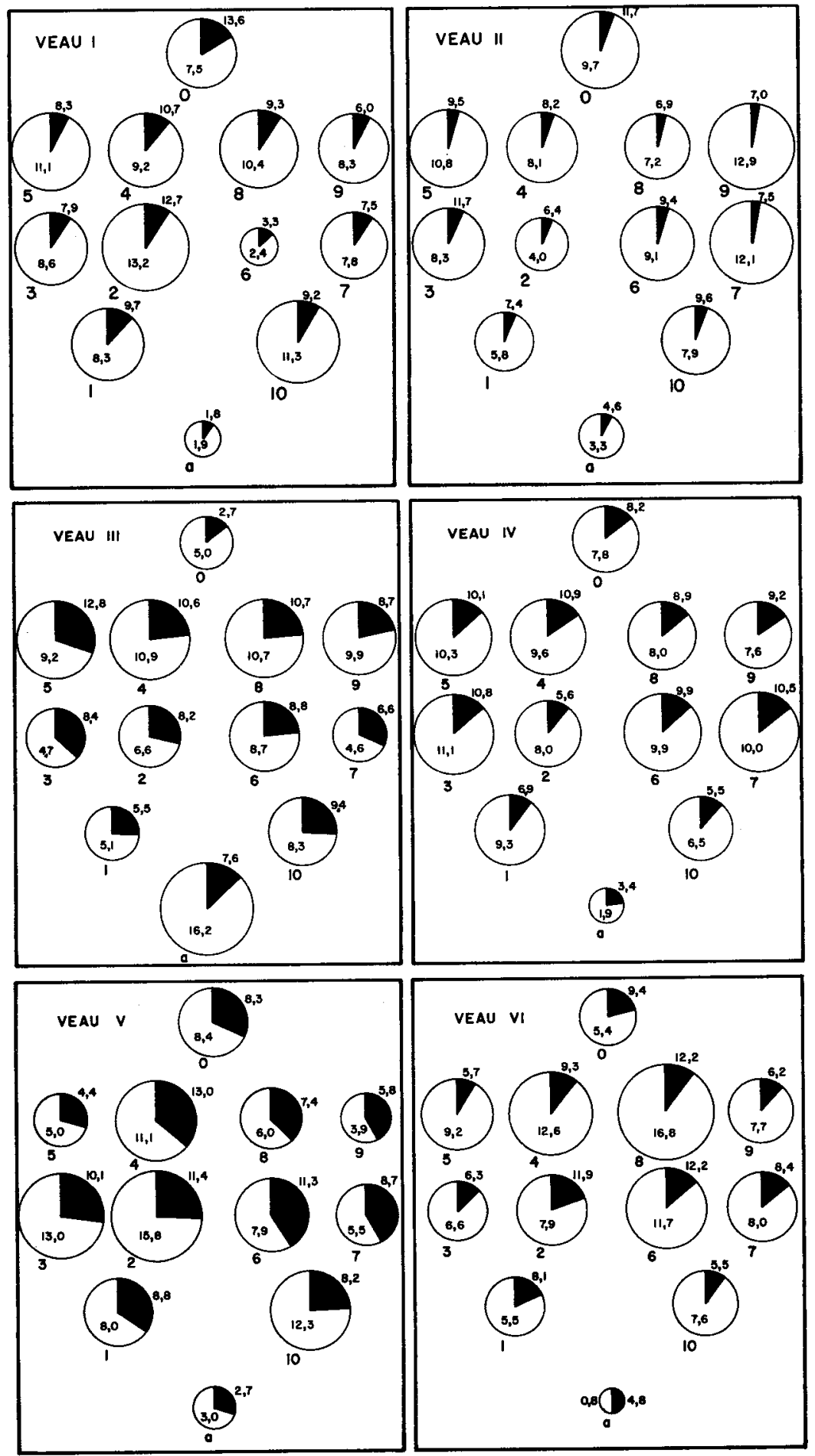


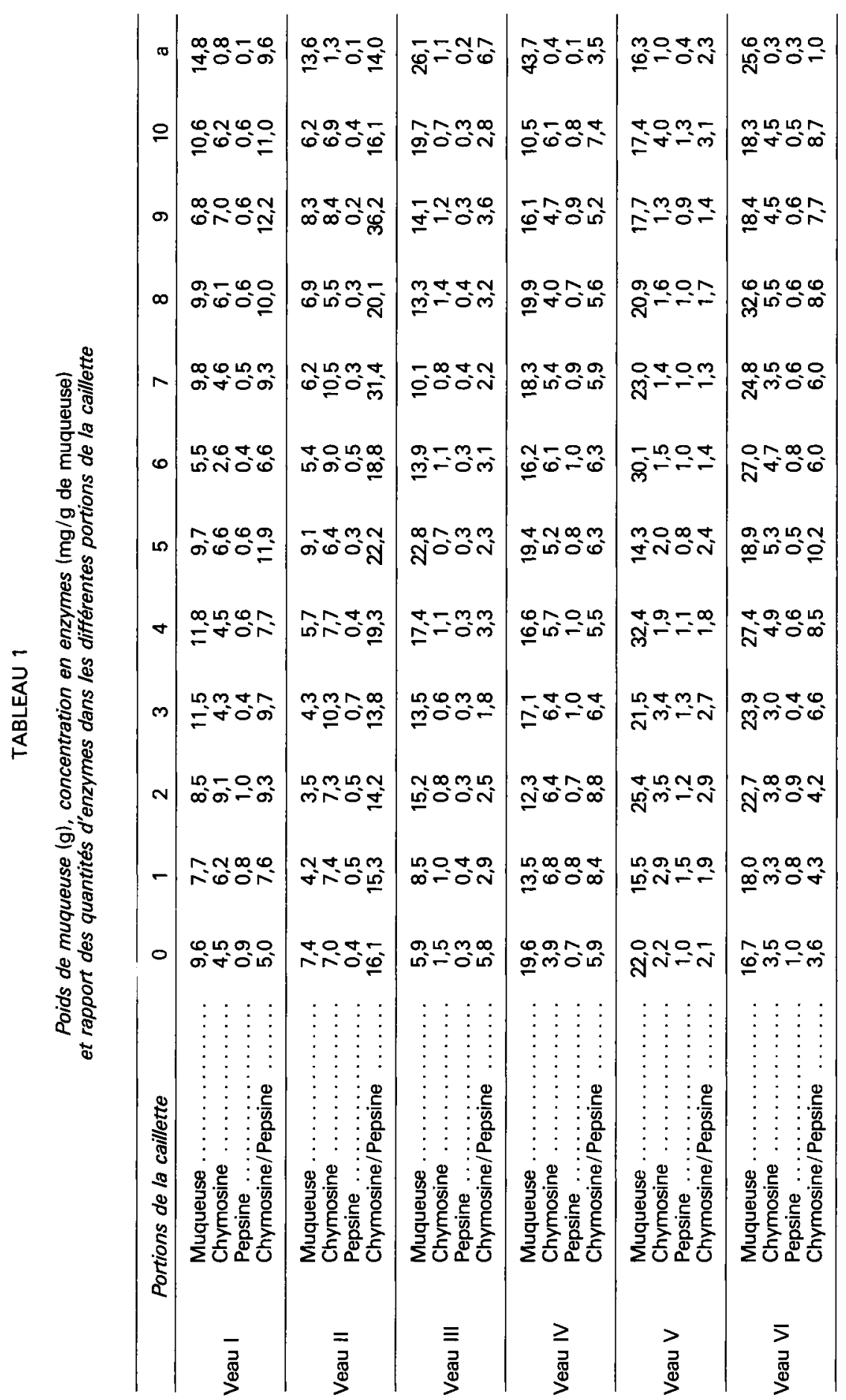




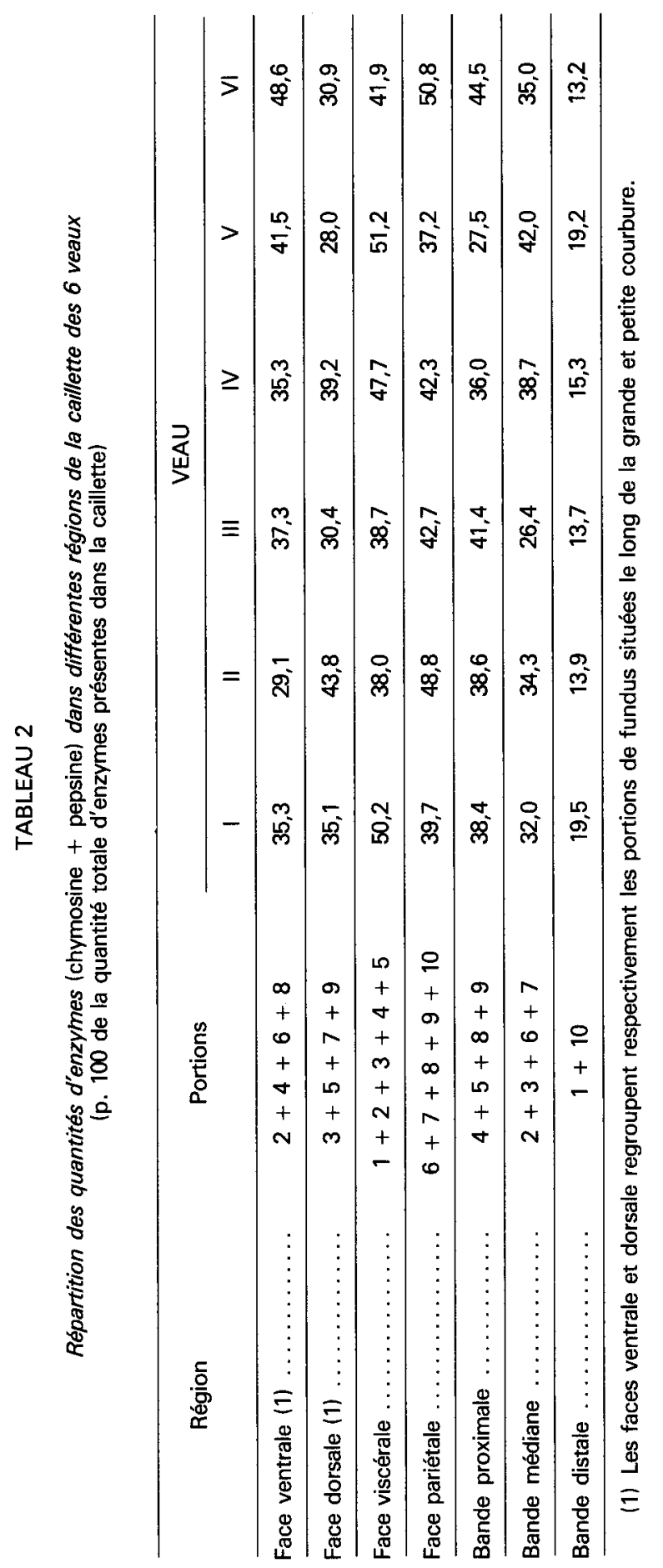


faces pariétale et viscérale ou suivant l'axe antéro-postérieur entre les bandes proximale, médiane et distale, ne semble pas varier notablement avec l'âge. En revanche, la part de la face ventrale (située le long de la grande courbure) augmente avec l'âge, aux dépens de celle de la face dorsale (située le long de la petite courbure) ; ainsi, la face ventrale rassemble en moyenne 45 p. 100 des enzymes totales de la caillette chez les animaux de 19 semaines, au lieu de $32 \mathrm{p}$. 100 chez les veaux nouveau-nés, les valeurs correspondantes pour la face dorsale étant de 29 et 39 p. 100 . Néanmoins, la part de la région constituée par les portions $3+7$ de cette dernière face varie dans des limites beaucoup plus étroites (de 18 p. 100 en moyenne à la naissance à 16 et 17 p. 100 à 10 et 19 semaines).

Le rapport chymosine/pepsine est en moyenne de $7,7 \pm 2,2$ dans la caillette entière (tabl. 3). A cet égard, aucune différence significative n'est observée entre celle-ci et les régions topographiques définies au tableau 2 ; il en est de même en comparant ces régions entre elles, par exemple les bandes proximale et distale, qui présentent pourtant l'écart maximum (respectivement $8,8 \pm 3,6$ et $7,3 \pm 2,2)$. La région dont l'évolution se rapproche le plus de celle de la caillette entière est encore celle constituée par les portions $3+7(7,7 \pm 2,8)$, suivie de la bande distale, avec respectivement plus de 80 et 60 p. 100 de chances pour que les différences observées soient dues au hasard.

\section{Discussion.}

Le découpage de la caillette que nous réalisons ménage une portion orificielle arbitraire dans le but $d^{\prime}$ isoler un type particulier de muqueuse stomacale : la muqueuse cardiale. Présente chez de nombreux monogastriques, elle a l'aspect d'une fine bandelette de quelques millimètres de largeur chez la chèvre (Hill, 1951) et encore plus réduite chez le mouton (Sommerville, 1956), interposée entre l'épithélium kératinisé des pré-estomacs et la muqueuse fundique. La muqueuse cardiale est décrite comme plus pauvre en glandes, de surcroît de nature muqueuse, donc non sécrétrice d'enzymes. Les concentrations en enzymes de la zone orificielle de nos animaux sont cependant identiques à celles des portions fundiques : il semble donc ne pas exister de muqueuse cardiale étendue chez le veau. Ce point est en accord avec nos observations histologiques sur des caillettes de veaux préruminants âgés de 19 à 20 semaines (Delansorne et al., résultats non publiés).

Les faibles concentrations enzymatiques généralement trouvées dans la muqueuse antrale, par rapport au reste de la caillette, confirment les résultats d'Andren, Bjoerck et Claesson (1980). Elles pourraient s'expliquer par une adsorption des enzymes sur le film muqueux de surface ou par la présence de suc résiduel reclus par capillarité dans les cryptes larges, profondes et ramifiées de l'antre. Toutefois, la sécrétion d'enzymes par l'antre gastrique elle-même, peut être envisagée. Ainsi chez le chien, des poches antrales produisent un suc visqueux et alcalin, contenant de la pepsine (Grossman et Marks, 1960). Par ailleurs, la présence de pepsinogène est mise en évidence par 


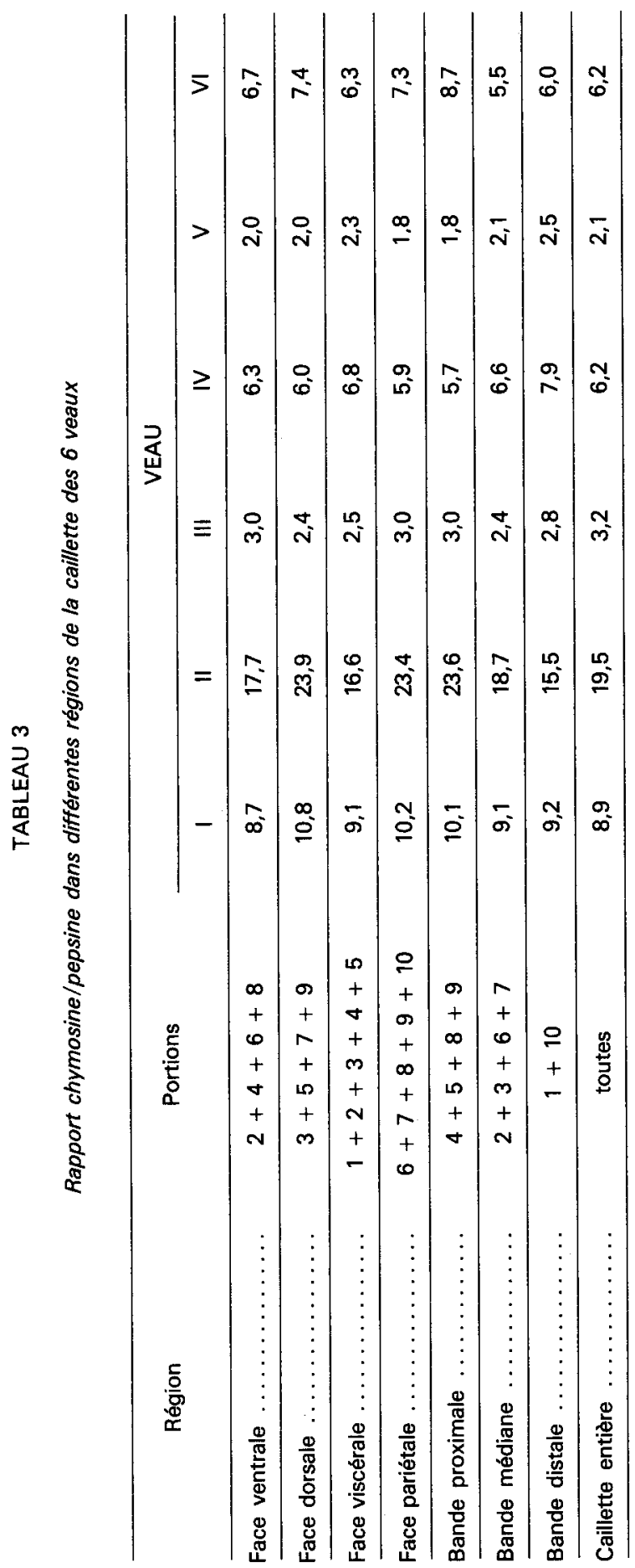


immunofluorescence dans les cellules des tubules glandulaires antraux chez I'homme (Samloff et Liebman, 1973), le cobaye (Zeitonn et al., 1972b), le chat et le chien (Liebman et Samloff, 1978). Chez le cobaye (Zeitonn et al., 1972a) et chez le chat (Lehy, communication personnelle), la microscopie électronique révèle la dualité sécrétoire (muqueuse et peptique) de ce type cellulaire au sein même de chaque grain de sécrétion.

Le fundus forme un réceptable en partie antérieure de la caillette. Au cours de la croissance, l'organe se développe et le sac fundique tend à s'agrandir et à se distinguer plus nettement de la partie distale plus étroite et plus musclée (Florentin, 1953). Malgré cela, aucune évolution avec l'âge ne peut être mise en évidence dans la répartition des contenus enzymatiques totaux entre les bandes proximale, médiane et distale. Cela pourrait être dû au fait que la découpe du fundus a toujours lieu en divisant par 3 la longueur totale de la grande courbure. La conformation interne présente des plis spiraux fundiques, indéformables, de faible épaisseur et de hauteur variable. Peu élevés à la naissance, ces plis peuvent atteindre plusieurs centimètres par la suite. Les plus hauts se trouvent en regard de la grande courbure. Leur taille décroît graduellement de part et d'autre pour s'annuler en regard de la petite courbure (l'espace toujours dénué de pli ainsi délimité constitue le sillon abomasique). L'augmentation des surfaces sécrétoires en zone ventrale explique donc l'accroissement avec l'âge de la part de cette zone dans le contenu enzymatique total de la caillette, au détriment de celle de la zone dorsale. Cependant, l'une des régions qui semblent le plus conserver la même représentativité par rapport à la caillette entière, en ce qui concerne les contenus enzymatiques et le rapport chymosine/pepsine, est constituée par la réunion des portions 3 et 7 communes à la face dorsale et à la bande médiane. Les poches gastriques que nous avons préparées jusqu'à présent (Guilloteau et Le Calve, 1977) occupent surtout cette région, en débordant éventuellement sur la bande distale. Elles devraient donc convenir à l'étude de l'évolution des sécrétions enzymatiques avec l'âge. Ce choix n'est cependant valable que dans la mesure où la croissance de la région ainsi définie est isométrique de celle de l'ensemble de la caillette.

La distinction classiquement appliquée à l'estomac du monograstrique entre réservoir fundique et pompe antropylorique (Bernier, 1980) trouve par cette étude une autre illustration en ce qui concerne l'abomasum du veau. Le fundus reçoit les aliments et sécrète les produits exocrines de la digestion gastrique, l'antre assure la vidange stomacale et une part de la régulation digestive, notamment endocrine par la forte densité de cellules à gastrine (Delansorne et al., résultats non publiés). Enfin, la chymosine est toujours plus abondante que la pepsine, conformément aux observations effectuées sur le veau (Garnot et al., 1974 ; Andren, Bjoerck et Claesson, 1980) et l'agneau (Guilloteau et al., cités par Thivend, Toullec et Guilloteau, 1980).

En conclusion, malgré les variations individuelles importantes, cette étude permet de mettre en évidence une augmentation avec l'âge des potentialités de sécrétion enzymatique de la face ventrale du fundus, au détriment de celles de la face dorsale. L'une des régions qui semblent le mieux convenir à la réalisation 
d'une poche abomasale susceptible de conserver longtemps la même représentativité par rapport à l'ensemble de la caillette, est constituée par la partie dorsale de la bande médiane (portions $3+7$ ); son extension éventuelle pourrait avoir lieu du côté de la bande distale. Pour étudier l'influence de l'âge, il est cependant nécessaire d'utiliser également d'autres techniques, telles que l'analyse enzymatique de la muqueuse de la caillette entière prélevée à l'abattage. De plus, la présente étude ne fournit pas d'informations quant à la répartition des potentialités de sécrétions d'eau et d'électrolytes. Ces observations devront donc être complétées grâce aux études d'histologie actuellement en cours.

Recu en août 1981

Accepté en décembre 1981

\section{Références}

ASH R. W., 1961. Acid secretion by the abomasum and its relation to the flow of food material in the sheep. J. Physiol., 156, 93-111.

ANDREN A., BJOERCK L., CLAESSON O., 1980. Quantification of chymosin (rennin) and pepsin in bovine abomasum by rocket immunoelectrophoresis. Swedish J. agric. Res., 10, 123-130.

BERNIER J. J., 1980. Physiologie de la digestion chez l'homme normal et l'opéré du tube digestif. Doin, Paris.

DOUILLARD R., RIBADEAU-DUMAS B., 1970. Détermination avec la caséine $x$ de l'activité protéolytique de la présure, de la pepsine de porc et des pepsines bovines. Bull. Soc. Chim. biol., 52, 1429-1445.

FLORENTIN M., 1953. Anatomie topographique des viscères abdominaux du bceuf et du veau. Rev. Méd. vét., 106, 657.

GARNOT P., THAPON J. L., MATHIEU C.-M., MAUBOIS J. L., RIBADEAU-DUMAS B., 1972. Determination of rennin and bovine pepsins in commercial rennets and abomasal juices, J. Dairy Sci., 55, 1641-1650.

GARNOT P., VALLES E., THAPON J. L., TOULLEC R., TOMASSONE R., RIBADEAU-DUMAS B., 1974. Influence of dietary proteins on rennin and pepsin content of preruminant calf vell. J. Dairy Res., 41, 19-23.

GROSSKOPF J. F. W., 1959. Some factors affecting the secretion of abomasal juice in young dairy calves. Onderst. J. vet. Res., 28, 133-141.

GROSSMANN M. I., MARKS I. N., 1960. Secretion of pepsinogen by the pyloric glands of the dog, with some observations on the histology of the gastric mucosa. Gastroenterology, 38, 343-352.

GUILLOTEAU P., LE CALVE J. L., 1977. Technique de réalisation d'une poche abomasale chez le veau en vue de l'obtention de suc gastrique pur. Ann. Biol. anim. Bioch. Biophys., 17. $1047-1060$.

HILL K. J., 1951. The glands of the mucous membrane of the goat abomasum. J. Anat. (Lond), 85, $216-220$.

HILL K. J., GREGORY R. A., 1951. The preparation of gastric pouches in the ruminant. Vet. Rec., 63, 647-652.

LIEBMAN W. M., SAMLOFF I. M., 1978. Immunochemical characterization and cellular localization of pepsinogens in cat and dog. J. Histochem. Cytochem., 26, 1115-1126.

RUSEV V., PETROV A., BOCHEVA E., 1971. Pecularities of rennet secretion in nursing calves in connection with the manner of feeding and milk temperature (en russe). Nautsh. Trud. Zootech. Fac., 22, 145-156. 
SAMLOFF I. M., LIEBMAN W. M., 1973. Cellular localization of the group II pepsinogens in human stomach and duodenum by immunofluorescence. Gastroenterology, 65, 36-42.

SOMMERVILLE R. I., 1956. The histology of the ovine abomasum and the relation of the globule leucocyte to nematode infestations. Austr. vet. J., 32, 237-240.

THIVEND P., TOULLEC R., GUILLOTEAU P., 1980. Digestive adaptation in the preruminant. In RUCKEBUSCH Y., THIVEND P., Digestive physiology and metabolism in ruminants, 561-586. Vth int. Symp. on Ruminant Physiology, 3-7 septembre 1979, Clermont-Ferrand (France) M.T.P. Press. Ltd, Lancaster.

WILLIAMS V. J., ROY J. H. B., GILLIES C. M., 1976. Milk substitute diet and abomasal secretion in the calf. Br. J. Nutr., 36, 317-335.

ZEITONN P., DUCLERT N., LIAUTAUD F., POTET F., 1972a. Localisation du pepsinogène associé aux mucines dans la muqueuse pylorique du cobaye. Ann. Anat. path. (Paris), 17, 327-333.

ZEITONN P., DUCLERT N., LIAUTAUD F., POTET F., ZYLBERBERG L., 1972b. Intracellular localization of pepsinogen in guinea pig pyloric mucosa by immunohistochemistry. Lab. Invest., 27.. 218-225. 